Research Paper

\title{
Prognostic Impact of Thrombospodin-2 (THBS2) Overexpression on Patients with Urothelial Carcinomas of Upper Urinary Tracts and Bladders
}

\author{
I-Wei Chang1,2, Chien-Feng Li33,4,5,6, Victor Chia-Hsiang Lin'2,7, Hong-Lin He1, Per-In Liang8, Wen-Jeng \\ $\mathrm{Wu}^{9,10,11}$, Ching-Chia $\mathrm{Li}^{9,10,11}$, Chun-Nung Huang, ${ }^{9,10 \bowtie}$ \\ 1. Department of Pathology, E-DA Hospital, I-Shou University, Kaohsiung, Taiwan; \\ 2. School of Medicine for International Students, I-Shou University, Kaohsiung, Taiwan; \\ 3. Department of Pathology, Chi-Mei Medical Center, Tainan, Taiwan; \\ 4. Department of Biotechnology, Southern Taiwan University of Science and Technology, Tainan, Taiwan; \\ 5. Graduate Institute of Medicine, College of Medicine, Kaohsiung Medical University, Kaohsiung, Taiwan; \\ 6. National Institute of Cancer Research, National Health Research Institutes, Tainan, Taiwan; \\ 7. Department of Urology, E-DA Hospital, I-Shou University, Kaohsiung, Taiwan; \\ 8. Department of Pathology, Kaohsiung Medical University Hospital, Kaohsiung Medical University, Kaohsiung, Taiwan; \\ 9. Department of Urology, School of Medicine, College of Medicine, Kaohsiung Medical University, Kaohsiung, Taiwan; \\ 10. Department of Urology, Kaohsiung Medical University Hospital, Kaohsiung, Taiwan: \\ 11. Department of Urology, Kaohsiung Municipal Ta-Tung Hospital, Kaohsiung, Taiwan. \\ $\square$ Corresponding author: Chun-Nung Huang, M.D., PhD., Department of Urology, Department of Pathology, Kaohsiung Medical University Hospital, \\ Kaohsiung, Taiwan. Email: cnhuang.uro@gmail.com.
}

(0) Ivyspring International Publisher. Reproduction is permitted for personal, noncommercial use, provided that the article is in whole, unmodified, and properly cited. See http://ivyspring.com/terms for terms and conditions.

Received: 2016.03.30; Accepted: 2016.05.31; Published: 2016.07.08

\begin{abstract}
Purpose: Urothelial carcinoma (UC) is a type of tumor, especially of the urinary bladder, that affects people worldwide. Clarification of its detailed tumor biology and discovery of potential targets for developing treatment strategies are imperative because of frequent recurrences and poor prognosis of advanced UCs. By data mining a published dataset of UC of bladder (UCB) transcriptome (GSE31684) from Gene Expression Omnibus, National Center of Biotechnology Information (GEO, NCBI), we identified that THBS2 was the most significantly upregulated gene among those related to structural molecule activity (GO:0005198). Therefore, we evaluated the clinical significance and prognostic impact of thrombospondin-2 (THBS2) protein, A.K.A. TSP2, which encoded by THBS2 gene.

Materials and Methods: THBS2 immunostaining was performed in 340 UCs of upper urinary tract (UC-UUTs) and 295 UCBs; subsequently, both groups were dichotomized into high- and low-expression subgroups. Moreover, statistical analyses were performed to correlate the association between THBS2 expression and clinicopathological parameters with two survival indexes: disease-specific survival (DSS) and metastasis-free survival (MeFS).

Results: High THBS2 immunoexpression was significantly associated with advanced primary tumor status, nodal metastasis, and vascular invasion in both UC-UUT and UCB groups (all $P \leq$ $.001)$. In addition, THBS2 overexpression was linked to adverse DSS and MeFS in univariate analyses and served as an independent prognosticator indicating poor outcomes in both groups in multivariate analyses.

Conclusion: THBS2 may play a crucial role in UC progression and may be a novel prognostic marker. Additional investigations to elucidate the molecular pathway are necessary for developing potential THBS2-targeted therapies for UCs.
\end{abstract}

Key words: prognosis, THBS2, thrombospondin-2, TSP2, urothelial carcinoma. 


\section{Introduction}

Urothelial carcinoma (UC), also known as transitional cell carcinoma, is the most common histological type of urinary system malignancy, especially in developed countries [1]. UC arises from the urothelium of the entire urinary tract, from the renal pelvis and ureter to the urinary bladder and urethra. The renal pelvis and ureter constitute the upper urinary tract (UUT), and the urinary bladder and urethra constitute the lower urinary tract (LUT). In contrast to UC of the bladder (UCB), the ninth most common malignancy [2], UC of the upper urinary tract (UC-UUT) is relatively uncommon and accounts for only $5 \%-10 \%$ of all UCs [3]. Although all UCs share some identical carcinogens, such as cigarette smoking and occupational exposure to aromatic amines [4-6], some diseases have a closer relationship with UC-UUT. For example, patients with Chinese herb nephropathy, endemic Balkans nephropathy, and analgesic nephropathy are more susceptible to UC-UUT than UCB [7-10]. Notably, UC-UUT is prevalent in Taiwan, especially in the endemic areas of blackfoot disease, which is caused by arsenic-contaminated water [11-13]. In addition, the gene expression profiles of both UC-UUT and UCB are similar [14] and their clinical behaviors are alike after balancing the stage and grade [15]. These evidences indicate that the carcinogenesis of UCs along the entire urinary tract may have, at least partly, a similar molecular pathway.

Structural proteins are those forming cellular ultrastructures and include fibrous proteins, globular proteins, motor proteins, and some subsets of membrane proteins. They contribute to extracellular matrix and cytoskeleton formation, cellular motility, and cell-cell adhesion [16]. Alterations of these proteins are crucial in the development of tumor invasion and metastasis in oncogenesis [17-19]. However, genes associated with structural molecules have not been systemically evaluated in UCs. By data mining a published dataset of UCB transcriptome (GSE31684) from Gene Expression Omnibus, National Center of Biotechnology Information (GEO, NCBI, Bethesda, MD, USA), we identified that THBS2 was the most significantly upregulated gene among those related to structural molecule activity (GO:0005198). THBS2 gene encodes thrombospondin-2 (THBS2, also known as TSP2). THBS2 is a disulfide-linked glycoprotein that mediates extracellular matrix assembly, cell-to-matrix interactions, degradation of matrix metalloproteinase (MMP)-2 and MMP-9, and inhibition of angiogenesis [20]. The expression of THBS2 and its prognostic value have been investigated in several cancers [21-28]. Nevertheless, to the best of our knowledge, no study has researched the expression of THBS2 in UCs. Hence, we focused on THBS2 as the target for the experiment detailed in the subsequent section.

\section{Materials and Methods}

\section{Data Mining of Transcriptomic Database from GEO to Identify the Most Altered Gene}

A transcriptomic dataset (GSE31684), comprising 93 radically resected UCB cases from GEO, NCBI, was selected for research; of these, 78 belonged to muscle-invasive tumors (pT2 to pT4) and 15 to non-muscle-invasive (pTa and pT1); 28 exhibited distant metastasis whereas 49 did not [29]. In this data set, GeneChip ${ }^{\circledR}$ Human Genome U133 Plus 2.0 array (Affymetrix, Santa Clara, CA, USA) was used for analysis. After downloading the raw data, Nexus Expression 3 statistical software (BioDiscovery, El Segundo, CA, USA) was used to analyze all probe sets without filtering or preselection. Moreover, under supervision, we inspected the statistical significance of each transcript by comparing the primary tumor invasiveness and the presence or absence of metastatic diseases. We performed functional profiling by using transcriptomes of muscle-infiltrative UCBs (pT2 to pT4) with metastases and those of non-muscle-infiltrative UCBs (pTa and pT1) without metastasis, targeting the transcriptomes associated with structural molecule activity (GO:0005198). Only genes showing significantly differential expression $(P<.01)$ were enrolled.

\section{Study Cohort of Patients and Specimens}

The study was approved by the Institutional Review Board of Chi-Mei Medical Center (10501005) and E-Da Hospital (EMRP-104-119). Our well-characterized study cohort comprised 635 consecutive patients with surgically resected UCs from 1996 to 2004 and included 295 patients with UCBs and 340 with UTT-UCs. Histopathological classifications other than UC and variants of UC were excluded. The criteria for inclusion and exclusion and treatment protocols were the same as previously described [30].

\section{Immunohistochemistry and Assessment}

Tissue sections were subjected to the routine procedures of deparaffinization, rehydration, and epitope retrieval and were subsequently incubated with an antibody against THBS2 (1:50, Rabbit polyclonal, Novus Biologicals, Littleton, CO, USA) for 1 hour. XXXX tissues with or without incubation of THBS2 antibody were run in parallel as positive and negative control, respectively. We used the $\mathrm{H}$ score method to evaluate the expression of THBS2 protein, which combines the intensity and percentage of 
immunostaining in the tumor cells to create an $\mathrm{H}$ score. The equation for assessing the $\mathrm{H}$ score is as follows: $\mathrm{H}$ score $=\Sigma P_{i}(i+1)$, where $P_{i}$ represents the percentage of stained tumor cells $(0 \%-100 \%)$ and $i$ represents the intensity of immunoreactivity $(0-3+)$. This formula yields a score in the range 100-400, where 100 signifies that $100 \%$ of the cancer cells are unreactive and 400 signifies that $100 \%$ of the cancer cells are strongly immunoreactive (3+).

\section{Statistical Analyses}

We used SPSS Version 14.0 (SPSS Inc., Chicago, IL, USA) for statistical analysis. The median $\mathrm{H}$ score of THBS2 immunoreactivity was applied as the cut-off value to classify our patient cohort into high- and low-expression subgroups for both the UC-UUT and UCB groups, respectively. We applied Pearson's chi-squared test to compare the association between THBS2 expression and miscellaneous critical categorical clinical and pathological parameters and Mann-Whitney U test for numerical parameters. Two prognostic indices, disease-specific survival (DSS) and metastasis-free survival (MeFS) intervals, were determined from the day of surgical excision to the day patient death or occurrence of metastatic disease. Patients lost to follow-up were excluded from the latest follow-up date. For univariate survival analyses, Kaplan-Meier survival curves, with comparison by using log-rank test, were utilized. Parameters demonstrating $P<.05$ in univariate analyses were included in multivariate tests by using Cox proportional hazards regression. For all statistical analyses, we used two-tailed tests of significance, and only $P<.05$ was judged to be statistically significant.

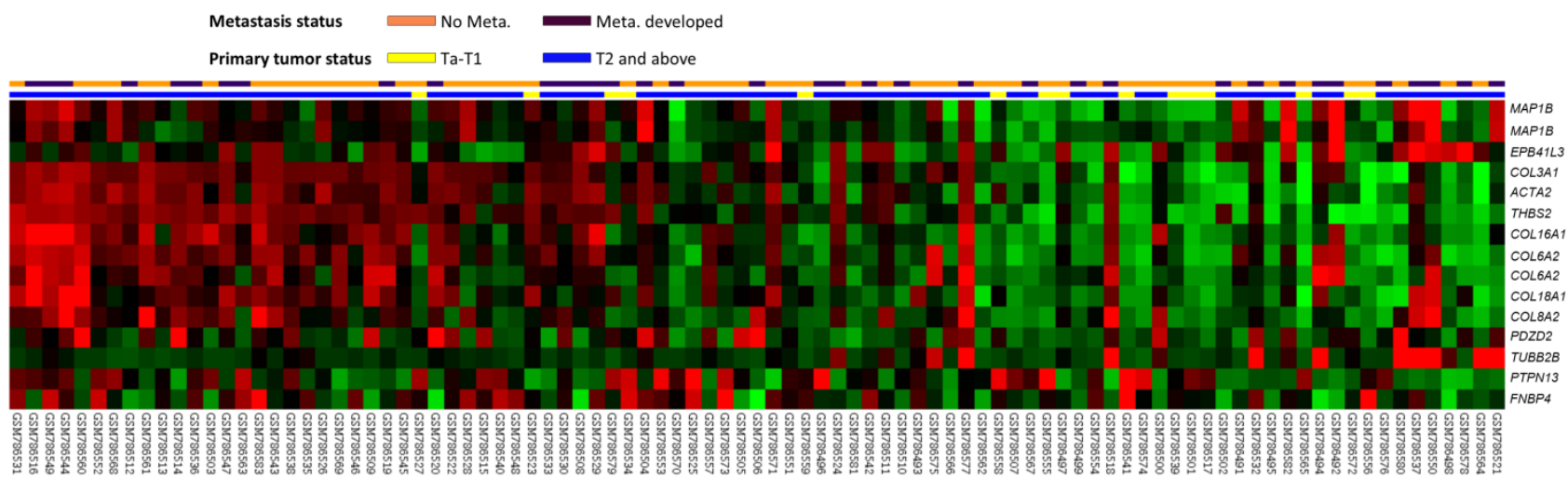

Figure 1. Analysis of gene expression in urothelial carcinoma of the urinary bladder by using a published transcriptome data set (GSE3 1684). Conducting a clustering analysis of genes by focusing on structural molecule activity (GO:0005198) revealed that THBS2 was one of the most significantly upregulated genes associated with a more advanced $\mathrm{pT}$ status and metastatic disease. Tissue specimens from cancers with distinct $\mathrm{p} T$ statuses are illustrated at the top of the heat map, and the expression levels of upregulated and downregulated genes are represented as a continuum of brightness of red or green, respectively. Specimens with unaltered mRNA expression are in black.

\section{Results}

\section{THBS2 as the Most Upregulated Gene among Those Belonging to Structural Molecule Activity (GO:0005 198)}

The gene expression profiling is presented in Fig. 1. By analyzing the published transcriptomic database of UCB (GSE31684) from GEO, 15 probes covering 13 genes related to structural molecule activity (GO:0005198) showed highly significant alteration $(P$ $<.01$ ), either upregulation or downregulation, associated with a more aggressive disease. When comparing muscle-infiltrative tumors (pT2 to pT4) to non-muscle-infiltrative tumors (pTa and pT1) and the presence to absence of distant metastasis, 11 genes, i.e., THBS2, COL6A2, COL3A1, MAP1B, COL16A1, ACTA2, EPB41L3, TUBB2B, COL8A2, COL18A1, and $P D Z D 2$, were upregulated to statistical significance (Table 1, all $P<.01$ ). Among them, THBS2 was the most significantly upregulated gene with 2.2182-fold $\log 2$ ratio by comparison between more advanced primary tumors (pT2-4) and less advanced ones ( $\mathrm{pTa}$ and pT1).

\section{Clinical and Pathological Characteristics of Both UC-UUT and UCB Groups}

As shown in Table 2, there was a male predominance $(n=216,73.2 \%)$ in the UCB group, in contrast to the lack of a gender predominance in the UC-UUT group (M/F, 46.5\%/53.5\%). In both groups, most patients were elderly (mean age $>65 \mathrm{y}$ ). Sixty-two of the 340 patients $(18.2 \%)$ with UC-UUT had multiple tumors; among them, 49 patients had synchronous tumors in both the renal pelvis and ureter. Most patients had muscle-infiltrating tumors (pT2-4, 46.8\% and $41.7 \%$ for UC-UUT and UCB groups, respectively). Similarly, most tumors were classified as having a high histopathological grade 
(83.5\% and $81 \%$ for UC-UUT and UCB groups, Vascular invasion and perineural invasion were respectively). Furthermore, 8.2\% $(\mathrm{n}=28)$ and 9.8\% (n $=29$ ) of the patients with UC-UUT and UCB, respectively, had lymph node metastasis at diagnosis. identified in $31.1 \%(\mathrm{n}=106)$ and $5.6 \%(\mathrm{n}=19)$ UC-UUTs as well as in $16.6 \%(\mathrm{n}=49)$ and $6.8 \%(\mathrm{n}=$ 20) UCBs.

Table 1. Summary of differentially expressed genes associated with structural molecule activity (GO:0005198) and showed positive associations to cancer invasiveness and metastasis in the transcriptome of urothelial carcinoma of urinary bladder (GSE31684).

\begin{tabular}{|c|c|c|c|c|c|c|c|}
\hline \multirow[t]{2}{*}{ Probe } & \multicolumn{2}{|c|}{$\begin{array}{l}\text { Comparing T2-4 } \\
\text { to Ta-T1 }\end{array}$} & \multicolumn{2}{|c|}{$\begin{array}{l}\text { Comparing Meta. } \\
\text { to Non-Meta.\# }\end{array}$} & \multirow[t]{2}{*}{$\begin{array}{l}\text { Gene } \\
\text { Symbol }\end{array}$} & \multirow[t]{2}{*}{ Biological Process } & \multirow[t]{2}{*}{ Molecular Function } \\
\hline & $\log$ ratio & p-value & $\log$ ratio & p-value & & & \\
\hline 203083_at & 2.2182 & $<0.0001$ & 0.9344 & 0.003 & THBS2 & cell adhesion & $\begin{array}{l}\text { calcium ion binding, heparin binding, protein } \\
\text { binding, structural molecule activity }\end{array}$ \\
\hline 209156_s_at & 2.1304 & $<0.0001$ & 0.9732 & 0.0021 & COL6A2 & $\begin{array}{l}\text { cell adhesion, cell-cell adhesion, extracellular } \\
\text { matrix organization and biogenesis, phosphate } \\
\text { transport }\end{array}$ & $\begin{array}{l}\text { extracellular matrix structural constituent, protein } \\
\text { binding, protein binding; bridging, structural } \\
\text { molecule activity }\end{array}$ \\
\hline 215076_s_at & 1.4494 & $<0.0001$ & 0.5177 & 0.0031 & COL3A1 & $\begin{array}{l}\text { circulation, organ morphogenesis, phosphate } \\
\text { transport }\end{array}$ & $\begin{array}{l}\text { extracellular matrix structural constituent, structural } \\
\text { molecule activity }\end{array}$ \\
\hline 226084_at & 1.2832 & $<0.0001$ & 0.9436 & $<0.0001$ & $M A P 1 B$ & $\begin{array}{l}\text { dendrite development, microtubule bundle } \\
\text { formation }\end{array}$ & protein binding, structural molecule activity \\
\hline 204345_at & 1.274 & $<0.0001$ & 0.5661 & 0.001 & COL16A1 & $\begin{array}{l}\text { cell adhesion, female pregnancy, } \\
\text { integrin-mediated signaling pathway, phosphate } \\
\text { transport }\end{array}$ & $\begin{array}{l}\text { integrin binding, protein binding, structural } \\
\text { molecule activity }\end{array}$ \\
\hline 200974_at & 1.2155 & 0.0002 & 0.6759 & 0.0087 & ACTA2 & & $\begin{array}{l}\text { ATP binding, nucleotide binding, protein binding, } \\
\text { structural molecule activity }\end{array}$ \\
\hline 212681_at & 1.0972 & 0.0001 & 1.2658 & $<0.0001$ & EPB41L3 & $\begin{array}{l}\text { cortical actin cytoskeleton organization and } \\
\text { biogenesis }\end{array}$ & $\begin{array}{l}\text { actin binding, binding, cytoskeletal protein binding, } \\
\text { protein binding, structural molecule activity }\end{array}$ \\
\hline 214023_x_at & 0.9093 & 0.0018 & 0.9548 & $<0.0001$ & TUBВ2B & $\begin{array}{l}\text { microtubule-based movement, microtubule-based } \\
\text { process, protein polymerization }\end{array}$ & $\begin{array}{l}\text { GTP binding, GTPase activity, nucleotide binding, } \\
\text { protein binding, structural constituent of } \\
\text { cytoskeleton, structural molecule activity }\end{array}$ \\
\hline 221900_at & 0.8558 & $<0.0001$ & 0.3936 & 0.0014 & COL8A2 & $\begin{array}{l}\text { cell adhesion, cell-cell adhesion, extracellular } \\
\text { matrix organization and biogenesis, phosphate } \\
\text { transport }\end{array}$ & $\begin{array}{l}\text { extracellular matrix structural constituent, protein } \\
\text { binding, protein binding; bridging, structural } \\
\text { molecule activity }\end{array}$ \\
\hline 213290_at & 0.832 & 0.0002 & 0.5142 & 0.004 & COL6A2 & $\begin{array}{l}\text { cell adhesion, cell-cell adhesion, extracellular } \\
\text { matrix organization and biogenesis, phosphate } \\
\text { transport }\end{array}$ & $\begin{array}{l}\text { extracellular matrix structural constituent, protein } \\
\text { binding, protein binding; bridging, structural } \\
\text { molecule activity }\end{array}$ \\
\hline 209082_s_at & 0.6536 & 0.0015 & 0.4516 & 0.0042 & COL18A1 & $\begin{array}{l}\text { angiogenesis, cell adhesion, endothelial cell } \\
\text { morphogenesis, extracellular matrix organization } \\
\text { and biogenesis, negative regulation of cell } \\
\text { proliferation, organ morphogenesis, phosphate } \\
\text { transport, positive regulation of apoptosis, } \\
\text { positive regulation of cell migration, positive } \\
\text { regulation of cell proliferation, visual perception }\end{array}$ & $\begin{array}{l}\text { extracellular matrix structural constituent, metal ion } \\
\text { binding, protein binding, structural molecule } \\
\text { activity, zinc ion binding }\end{array}$ \\
\hline 209493_at & 0.4147 & 0.0095 & 0.436 & 0.0003 & PDZD2 & cell adhesion & protein binding, structural molecule activity \\
\hline 214577_at & 0.3773 & 0.0029 & 0.3943 & $<0.0001$ & $M A P 1 B$ & $\begin{array}{l}\text { dendrite development, microtubule bundle } \\
\text { formation }\end{array}$ & protein binding, structural molecule activity \\
\hline
\end{tabular}

\#, Meta., distal metastasis developed during follow-up; Non-Meta.: no metastatic event developed.

Table 2. Correlations between THBS2 Expression and other important clinicopathological parameters in urothelial carcinomas.

\begin{tabular}{|c|c|c|c|c|c|c|c|c|c|}
\hline \multirow[t]{3}{*}{ Parameter } & \multirow[t]{3}{*}{ Category } & \multicolumn{4}{|c|}{ Upper Urinary Tract Urothelial Carcinoma } & \multicolumn{4}{|c|}{ Urinary Bladder Urothelial Carcinoma } \\
\hline & & \multirow[t]{2}{*}{ Case No. } & \multicolumn{2}{|c|}{ THBS2 Expression } & \multirow[t]{2}{*}{ p-value } & \multirow[t]{2}{*}{ Case No. } & \multicolumn{2}{|c|}{ THBS2 Expression } & \multirow[t]{2}{*}{ p-value } \\
\hline & & & Low & High & & & Low & High & \\
\hline \multirow[t]{2}{*}{ Gender\& } & Male & 158 & 75 & 83 & 0.384 & 216 & 110 & 106 & 0.534 \\
\hline & Female & 182 & 95 & 87 & & 79 & 37 & 42 & \\
\hline Age (years)"\# & & 340 & $65.94+/-10.01$ & $65.22+/-9.69$ & 0.214 & 295 & $66.04+/-12.67$ & $66.05+/-11.78$ & 0.704 \\
\hline \multirow[t]{3}{*}{ Tumor location\& } & Renal pelvis & 141 & 70 & 71 & 0.685 & - & - & - & - \\
\hline & Ureter & 150 & 78 & 72 & & - & - & - & - \\
\hline & Renal pelvis \& ureter & 49 & 22 & 27 & & - & - & - & - \\
\hline \multirow[t]{2}{*}{ Multifocality\& } & Single & 278 & 142 & 136 & 0.339 & - & - & - & - \\
\hline & Multifocal & 62 & 28 & 34 & & - & - & - & - \\
\hline \multirow[t]{3}{*}{ Primary tumor $(\mathrm{pT})$ \& } & $\mathrm{pTa}$ & 89 & 63 & 26 & $<0.001^{*}$ & 84 & 68 & 16 & $<0.001^{*}$ \\
\hline & pT1 & 92 & 50 & 42 & & 88 & 46 & 42 & \\
\hline & pT2-pT4 & 159 & 57 & 102 & & 123 & 33 & 90 & \\
\hline \multirow[t]{2}{*}{ Nodal metastasis\& } & Negative (pN0) & 312 & 163 & 149 & $<0.001^{*}$ & 266 & 141 & 125 & $0.001^{*}$ \\
\hline & Positive (pN1-pN3) & 28 & 7 & 21 & & 29 & 6 & 23 & \\
\hline \multirow[t]{2}{*}{ Histological grade \& } & Low grade & 56 & 34 & 22 & 0.079 & 56 & 44 & 12 & $0.001^{*}$ \\
\hline & High grade & 284 & 136 & 148 & & 239 & 103 & 136 & \\
\hline \multirow[t]{2}{*}{ Vascular invasion\& } & Absent & 234 & 132 & 102 & $<0.001^{*}$ & 246 & 134 & 112 & $<0.001^{*}$ \\
\hline & Present & 106 & 38 & 68 & & 49 & 13 & 36 & \\
\hline \multirow[t]{2}{*}{ Perineural invasion\& } & Absent & 321 & 166 & 155 & $0.009^{*}$ & 275 & 141 & 134 & 0.066 \\
\hline & Present & 19 & 4 & 15 & & 20 & 6 & 14 & \\
\hline $\begin{array}{l}\text { Mitotic rate (per } 10 \\
\text { high power fields)\# }\end{array}$ & & 340 & $11.59+/-12.31$ & $13.02+/-12.24$ & 0.072 & 295 & $12.10+/-14.67$ & $16.70+/-13.03$ & $<0.001^{*}$ \\
\hline
\end{tabular}

\&, Chi-Square test; \#, Mann-Whitney U test; * Statistically significant. 


\section{Association Between THBS2 Immunoreactivity and Critical Clinical and Pathological Parameters}

Table 2 shows that high THBS2 expression was significantly associated with a more advanced primary tumor status (pT status, Fig. 2), lymph node metastasis at diagnosis, and vascular invasion in both UC-UUT and UCB groups (all $P \leq .001$ ). THBS2 overexpression was significantly associated with perineural invasion in UC-UUTs $(P=.009)$ as well as a high histological grade and higher mitotic activity in UCBs (both $P \leq .001$ ).

\section{Survival Analyses for UC-UUT and UCB Groups}

The survival analyses are summarized in Table 3 and Table 4 . In univariate analysis, advanced primary tumor status $(\mathrm{pT})$, nodal metastatic disease, high histopathological grade, and vascular and perineural invasions were significantly associated with shorter DSS and MeFS intervals in both UC-UUT and UCB groups (all $P<.05$ ). Multifocal tumors were significantly linked to both worse DSS $(P=.0042)$ and MeFS $(P=.0196)$, whereas tumor location was negatively associated with DSS $(P=.0120)$ only in the UC-UUT group. High mitotic activity (more than 10 mitoses per 10 high power fields) significantly correlated with both shorter DSS $(P=.0001)$ and MeFS $(P=.0002)$ intervals in the UCB group. In multivariate analysis of the UC-UUT group, multifocal tumors, nodal metastasis, high histological grade, and perineural invasion were predictive of both adverse DSS and MeFS (all $P<.05$ ). Higher $p T$ status and vascular invasion were independent prognosticators for only DSS and MeFS, respectively (both $P<.05$ ). Multivariate analysis for UCB group indicated that advanced tumor status was an independent prognostic factor for both adverse DSS and MeFS $(P<.001$ and $P=.023$, respectively). Perineural invasion and high mitotic rate only predicted dismal DSS $(P=.009$ and $P=.037$, respectively), whereas lymph node metastasis only predicted unfavorable MeFS $(P=.021)$.

\section{Prognostic Impact of THBS2 Expression on UC-UUTs and UCBs}

In univariate analysis, high THBS2 expression was significantly associated with poor DSS and MeFS in both the UC-UUT and UCB groups (Table 3 and Table 4; Fig. 3, all $P<0.0001)$. In multivariate analysis, THBS2 overexpression was an independent negative prognosticator that predicted unfavorable outcomes in both UC groups (all $P<0.05)$.
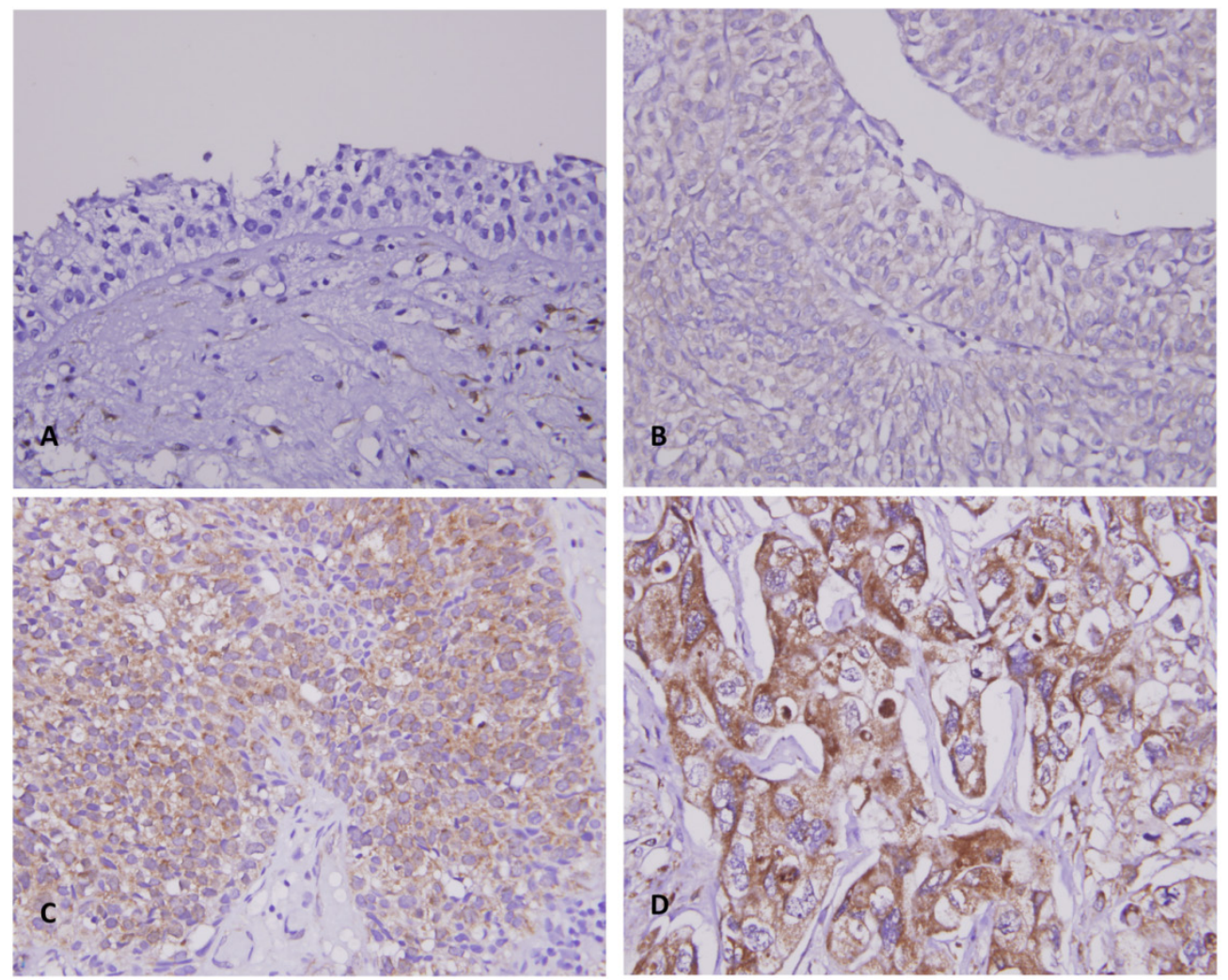

Figure 2. THBS2 immunostain on representative tissue sections revealed a stepwise increase of THBS2 immunoreactivity from the normal urothelial epithelium (A) to noninvasive papillary (PTa) (B), superficially infiltrating ( $\mathrm{PT1}$ ) (C), and muscle invasive (PT2-pT4) urothelial carcinomas (D). 
Table 3. Univariate log-rank and multivariate analyses for Disease-specific and Metastasis-free Survivals in upper urinary tract urothelial carcinoma.

\begin{tabular}{|c|c|c|c|c|c|c|c|c|c|c|c|c|}
\hline \multirow[t]{3}{*}{ Parameter } & \multirow[t]{3}{*}{ Category } & \multirow{3}{*}{$\begin{array}{l}\text { Case } \\
\text { No. }\end{array}$} & \multicolumn{5}{|c|}{ Disease-specific Survival } & \multicolumn{5}{|c|}{ Metastasis-free Survival } \\
\hline & & & \multicolumn{2}{|c|}{$\begin{array}{l}\text { Univariate } \\
\text { analysis }\end{array}$} & \multicolumn{3}{|c|}{ Multivariate analysis } & \multicolumn{2}{|c|}{$\begin{array}{l}\text { Univariate } \\
\text { analysis }\end{array}$} & \multicolumn{3}{|c|}{ Multivariate analysis } \\
\hline & & & $\begin{array}{l}\text { No. of } \\
\text { event }\end{array}$ & p-value & R.R. & 95\% C.I. & p-value & $\begin{array}{l}\text { No. of } \\
\text { event }\end{array}$ & $\mathrm{p}$-value & R.R. & 95\% C.I. & $\mathrm{p}$-value \\
\hline \multirow[t]{2}{*}{ Gender } & Male & 158 & 28 & 0.9301 & - & - & - & 32 & 0.7904 & - & - & - \\
\hline & Female & 182 & 33 & & - & - & - & 38 & & - & - & - \\
\hline \multirow[t]{2}{*}{ Age (years) } & $<65$ & 138 & 26 & 0.8660 & - & - & - & 30 & 0.8470 & - & - & - \\
\hline & $\geq 65$ & 202 & 35 & & - & - & - & 40 & & - & - & - \\
\hline \multirow[t]{3}{*}{ Tumor side } & Right & 177 & 34 & 0.7188 & - & - & - & 38 & 0.3074 & - & - & - \\
\hline & Left & 154 & 26 & & - & - & - & 32 & & - & - & - \\
\hline & Bilateral & 9 & 1 & & - & - & - & 0 & & - & - & - \\
\hline \multirow[t]{3}{*}{ Tumor location } & Renal pelvis & 141 & 24 & $0.0120^{*}$ & 1 & - & 0.976 & 31 & 0.0659 & - & - & - \\
\hline & Ureter & 150 & 22 & & 0.940 & $0.500-1.767$ & & 25 & & - & - & - \\
\hline & Renal pelvis \& ureter & 49 & 15 & & 1.140 & $0.319-4.074$ & & 14 & & - & - & \\
\hline \multirow[t]{2}{*}{ Multifocality } & Single & 273 & 48 & $0.0042^{*}$ & 1 & - & $0.012^{*}$ & 52 & $0.0196^{*}$ & 1 & - & $0.006^{*}$ \\
\hline & Multifocal & 62 & 18 & & 2.729 & $1.247-5.973$ & & 18 & & 2.175 & $1.254-3.773$ & \\
\hline \multirow[t]{3}{*}{ Primary tumor $(\mathrm{pT})$} & pTa & 89 & 2 & $<0.0001^{*}$ & 1 & - & $0.040^{*}$ & 4 & $<0.0001^{*}$ & 1 & - & 0.228 \\
\hline & pT1 & 92 & 9 & & 3.086 & $0.656-14.514$ & & 15 & & 2.712 & $0.877-8.383$ & \\
\hline & pT2-pT4 & 159 & 50 & & 4.580 & 1.003-20.919 & & 51 & & 2.479 & $0.770-7.976$ & \\
\hline \multirow[t]{2}{*}{ Nodal metastasis } & Negative (pN0) & 312 & 42 & $<0.0001^{*}$ & 1 & - & $<0.001^{*}$ & 55 & $<0.0001^{*}$ & 1 & - & $0.002^{*}$ \\
\hline & Positive (pN1-pN3) & 28 & 19 & & 4.935 & $2.681-9.085$ & & 15 & & 2.623 & $1.578-4.847$ & \\
\hline \multirow[t]{2}{*}{ Histological grade } & Low grade & 56 & 4 & $0.0171^{*}$ & 1 & - & $0.026^{*}$ & 3 & $0.0019^{*}$ & 1 & - & $0.126^{*}$ \\
\hline & High grade & 284 & 57 & & 3.099 & $1.144-8.395$ & & 67 & & 2.862 & $1.420-11.425$ & \\
\hline \multirow[t]{2}{*}{ Vascular invasion } & Absent & 234 & 24 & $<0.0001^{*}$ & 1 & - & 0.084 & 26 & $<0.0001^{*}$ & 1 & - & $0.001^{*}$ \\
\hline & Present & 106 & 37 & & 1.691 & $0.932-3.068$ & & 44 & & 2.643 & $1.456-4.130$ & \\
\hline \multirow[t]{2}{*}{ Perineural invasion } & Absent & 321 & 50 & $<0.0001^{*}$ & 1 & - & $0.001^{*}$ & 61 & $<0.0001^{*}$ & 1 & - & $0.029^{*}$ \\
\hline & Present & 19 & 11 & & 3.375 & $1.608-7.084$ & -- & 9 & & 2.282 & $1.089-4.784$ & \\
\hline \multirow{2}{*}{$\begin{array}{l}\text { Mitotic rate (per } 10 \\
\text { high power fields) }\end{array}$} & $<10$ & 173 & 27 & 0.1268 & - & - & - & 30 & 0.0581 & - & - & - \\
\hline & $>=10$ & 167 & 34 & & - & - & - & 40 & & - & - & - \\
\hline \multirow[t]{2}{*}{ THBS2 expression } & Low & 170 & 14 & $<0.0001^{*}$ & 1 & - & $0.017^{*}$ & 17 & $<0.0001^{*}$ & 1 & - & $0.002^{*}$ \\
\hline & High & 170 & 47 & & 2.133 & $1.146-3.969$ & & 53 & & 2.471 & $1.401-4.357$ & \\
\hline
\end{tabular}

* Statistically significant.

Table 4. Univariate log-rank and multivariate analyses for Disease-specific and Metastasis-free Survivals in urinary bladder urothelial carcinoma.

\begin{tabular}{|c|c|c|c|c|c|c|c|c|c|c|c|c|}
\hline \multirow[t]{3}{*}{ Parameter } & \multirow[t]{3}{*}{ Category } & \multirow{3}{*}{ Case No. } & \multicolumn{5}{|c|}{ Disease-specific Survival } & \multicolumn{5}{|c|}{ Metastasis-free Survival } \\
\hline & & & \multicolumn{2}{|c|}{ Univariate analysis } & \multicolumn{3}{|c|}{ Multivariate analysis } & \multicolumn{2}{|c|}{$\begin{array}{l}\text { Univariate } \\
\text { analysis }\end{array}$} & \multicolumn{3}{|c|}{ Multivariate analysis } \\
\hline & & & $\begin{array}{l}\text { No. of } \\
\text { event }\end{array}$ & p-value & R.R. & 95\% C.I. & p-value & $\begin{array}{l}\text { No. of } \\
\text { event }\end{array}$ & p-value & R.R. & 95\% C.I. & p-value \\
\hline \multirow[t]{2}{*}{ Gender } & Male & 216 & 41 & 0.4906 & - & - & - & 61 & 0.2745 & - & - & - \\
\hline & Female & 79 & 11 & & - & - & - & 16 & & - & - & - \\
\hline \multirow[t]{2}{*}{ Age (years) } & $<65$ & 121 & 17 & 0.1315 & - & - & - & 32 & 0.8786 & - & - & - \\
\hline & $\geq 65$ & 174 & 35 & & - & - & - & 45 & & & & \\
\hline \multirow[t]{3}{*}{ Primary tumor (pT) } & pTa & 84 & 1 & $<0.0001^{*}$ & 1 & - & $<0.001^{*}$ & 4 & $<0.0001^{*}$ & 1 & - & $0.023^{*}$ \\
\hline & pT1 & 88 & 9 & & 4.203 & $0.463-38.147$ & & 23 & & 3.326 & $0.998-11.080$ & \\
\hline & pT2-pT4 & 123 & 42 & & 18.697 & $2.142-163.230$ & & 50 & & 5.159 & $1.528-17.418$ & \\
\hline \multirow[t]{2}{*}{ Nodal metastasis } & Negative ( $\mathrm{pN} 0)$ & 266 & 41 & $0.0001^{*}$ & 1 & - & 0.550 & 61 & $<0.0001^{*}$ & 1 & - & $0.021^{*}$ \\
\hline & $\begin{array}{l}\text { Positive } \\
\text { (pN1-pN3) }\end{array}$ & 29 & 11 & & 1.242 & $0.610-2.530$ & & 16 & & 2.050 & $1.114-3.773$ & \\
\hline \multirow[t]{2}{*}{ Histological grade } & Low grade & 56 & 2 & $0.0016^{*}$ & 1 & - & 0.854 & 5 & $0.0007^{*}$ & 1 & - & 0.924 \\
\hline & High grade & 239 & 50 & & 1.158 & $0.243-5.518$ & & 72 & & 1.052 & $0.367-3.015$ & \\
\hline \multirow[t]{2}{*}{ Vascular invasion } & Absent & 246 & 37 & $0.0010^{*}$ & 1 & - & 0.181 & 54 & $<0.0001^{*}$ & 1 & - & 0.665 \\
\hline & Present & 49 & 15 & & 0.629 & $0.319-1.240$ & & 23 & & 1.141 & $0.630-2.063$ & \\
\hline \multirow[t]{2}{*}{ Perineural invasion } & Absent & 275 & 44 & $<0.0001^{*}$ & 1 & - & $0.009^{*}$ & 67 & $0.0003^{*}$ & 1 & - & 0.085 \\
\hline & Present & 20 & 8 & & 3.004 & $1.313-6.874$ & & 10 & & 1.886 & $0.916-3.881$ & \\
\hline \multirow{2}{*}{$\begin{array}{l}\text { Mitotic rate (per } 10 \\
\text { high power fields) }\end{array}$} & $<10$ & 139 & 12 & $0.0001^{*}$ & 1 & - & $0.037^{*}$ & 23 & $0.0002^{*}$ & 1 & - & 0.086 \\
\hline & $>=10$ & 156 & 40 & & 2.054 & $1.044-4.039$ & & 54 & & 1.572 & $0.938-2.635$ & \\
\hline \multirow[t]{2}{*}{ THBS2 expression } & Low & 147 & 10 & $<0.0001^{*}$ & 1 & - & $0.033^{*}$ & 18 & $<0.0001^{*}$ & 1 & - & $0.004^{*}$ \\
\hline & High & 148 & 42 & & 2.204 & $1.065-4.562$ & & 59 & & 2.275 & $1.307-3.960$ & \\
\hline
\end{tabular}

* Statistically significant. 

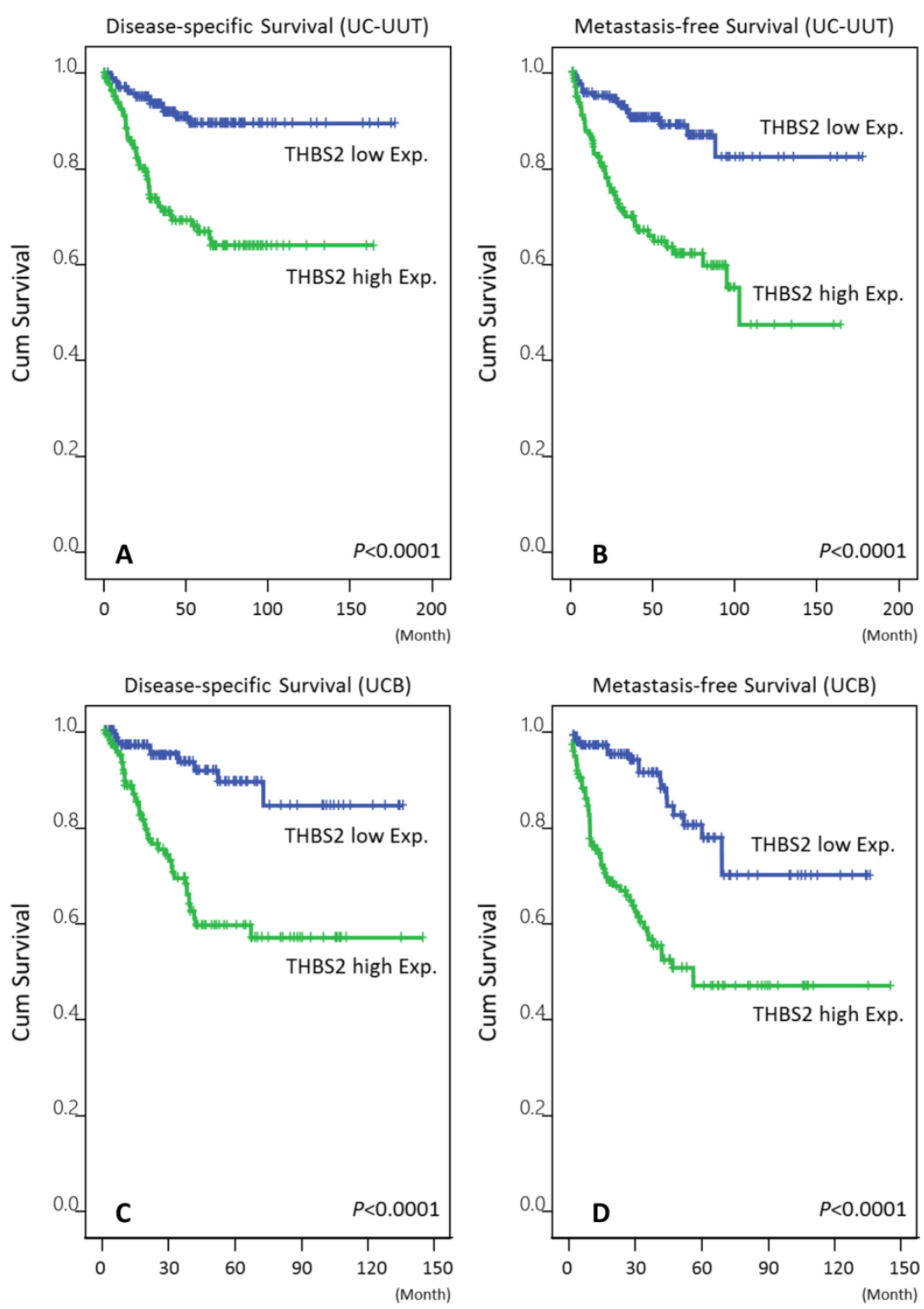

Figure 3. Kaplan-Meier plots demonstrated the extremely significant prognostic value of THBS2 expression for disease-specific survival (DSS) and metastasis-free survival (MeFS) rates in the UC-UUT (A and $\mathbf{B}$ for DSS and MeFS, respectively) and UCB (C and D for DSS and MeFS, respectively) groups (all $P<.0001$ ).

\section{Discussion}

Urothelial carcinoma is a cancer with high recurrent rate and the 5-year overall survival is still unsatisfactory for advanced disease in spite of the introduction of adjuvant chemotherapy [31]. Therefore, it is mandatory for scientists and physicians to develop more effective therapeutic strategies for high-risk patients.

THBS2 belongs to the thrombospondin (THBS/TSP) family of five calcium-binding matricellular glycoproteins, THBS1-THBS5. They are classified into two subgroups, namely trimeric and pentameric proteins, according to the oligomerization state and domain structure. THBS1 and THBS2 are trimeric proteins, whereas the others are pentameric
[32]. THBS1 and THBS2 have similar molecular structures and share $85 \%$ amino acid conservation and therefore have similar binding domains. Among the THBS/TSP family of proteins, only THBS1 and THBS2 contain the three type I properdin-like repeats, which contribute to the inhibition of angiogenesis [32]. THBS2 interacts with various cell-surface receptors, growth factors, cytokines, and proteases to modulate cell-matrix adhesion, motility, chemotaxis, wound healing, angioinhibition, among others [33]. It also participates in tumorigenesis, mainly by inhibiting angiogenesis and by negatively regulating MMP-2 and MMP-9 [34]. Therefore, THBS2 is usually considered to a tumor-suppressive gene. However, studies investigating THBS2 expression in various tumors have yielded inconsistent results. In a 
colorectal cancer study, THBS2 gene expression was negatively correlated with liver metastasis $(P=.02)$ and M1 status $(P=.03)$ [21]; high THBS2 immunoreactivity was not only associated with tumor response to neoadjuvant chemoradiotherapy but also with an independent and good prognostic factor for DSS and MeFS in patients with rectal cancer [28]. In one pancreatic cancer cell line study, the invasive property of the transfectants overexpressing THBS2 transcripts and protein was significantly suppressed $(P<.05)$ [22]. THBS2 gene downregulation was also observed in prostate cancer tissues and cell lines [25]. In addition, overexpression of THBS2 also proved to be a favorable prognostic factor for overall survival in patients with gastric cancer [26]. By contrast, in Chijiwa, et al., THBS2 transcriptional levels were significantly higher in lung adenocarcinoma compared with normal pulmonary tissues $(P<.0001)$ and were significantly higher in tumors with more advanced $\mathrm{T}$ statuses and stages compared with early cancers $(P=.0179$ for T3/4 to T1/2; $P=.0136$ for stages II/III to I) [23]. In the same study, THBS2 gene upregulation predicted poor overall survival $(P=$ .0139) [23]. In a recent study by Naumnik, et al., the serum concentration of THBS2 was significantly higher in patients with non-small cell lung cancer compared with healthy controls $(P=.002)$; in addition, high serum concentration of THBS2 correlated with shorter survival $(P=.007)$ [27]. The conflict maybe due to the divergent role of the same molecule in the differing microenvironments of various cancers. The current work is the first investigating the clinical correlation of THBS2 expression in both UCB and UC-UUT. The oncogenic property of THBS2 on UC maybe related to a mechanism other than the antiangiogenic effect.

\section{Conclusion}

The present study revealed overexpression of THBS2 played a significant role in tumor progression of UC. Overexpression of THBS2 was not only significantly associated with aggressive clinicopathological parameters but was also an independent poor prognostic biomarker predicting shorter DSS and MeFS in both UC-UUT and UCB. Additional investigations to elucidate the molecular pathway are necessary for developing potential THBS2-targeted therapies for both groups of UCs, similar to how we have previously developed hopeful molecular targets for UCs [35-41].

\section{Acknowledgements}

This study was supported by grants from Kaohsiung Medical University "Aim for the Top Universities" (KMU-TP104E31, KMU-TP104G00,
KMU-TP104G01, KMU-TP104G04), the health and welfare surcharge of tobacco products, Ministry of Health and Welfare (MOHW105-TDU-B-212-134007), Ministry of Science and Technology (MOST103-2314-B-037-059-MY3), and Kaohsiung Medical University Hospital (KMUH101-1R46, KMUH103-3M37). The present work was also supported by grants from E-DA Hospital to I-W Chang (EDAHP105062).

\section{Competing Interests}

No conflicts of interest to be declared.

\section{References}

1. Eble JN, Sauter G, Epstein JI, Sesterhenn IA. World Health Organization Classification of Tumours. Pathology and Genetics of Tumours of the Urinary System and Male Genital Organs. Lyon, France: International Agency for Research on Cancer (IARC) press; 2004.

2. Ploeg M, Aben KK, Kiemeney LA. The present and future burden of urinary bladder cancer in the world. World J Urol. 2009;27:289-93.

3. Roupret M, Zigeuner R, Palou J, et al. European guidelines for the diagnosis and management of upper urinary tract urothelial cell carcinomas: 2011 update. Eur Urol. 2011;59:584-94.

4. McLaughlin JK, Silverman DT, Hsing AW, et al. Cigarette smoking and cancers of the renal pelvis and ureter. Cancer Res. 1992;52:254-7.

5. Pommer W, Bronder E, Klimpel A, Helmert U, Greiser E, Molzahn M. Urothelial cancer at different tumour sites: role of smoking and habitual intake of analgesics and laxatives. Results of the Berlin Urothelial Cancer Study. Nephrol Dial Transplant. 1999;14:2892-7.

6. Shinka $T$, Miyai M, Sawada $Y$, Inagaki $T$, Okawa $T$. Factors affecting the occurrence of urothelial tumors in dye workers exposed to aromatic amines. Int J Urol. 1995;2:243-8

7. Debelle FD, Vanherweghem JL, Nortier JL. Aristolochic acid nephropathy: a worldwide problem. Kidney Int. 2008;74:158-69.

8. Dragicevic D, Djokic M, Pekmezovic T, et al. Survival of patients with transitional cell carcinoma of the ureter and renal pelvis in Balkan endemic nephropathy and non-endemic areas of Serbia. BJU Int. 2007;99:1357-62.

9. Laing C, Hamour S, Sheaff M, Miller R, Woolfson R. Chinese herbal uropathy and nephropathy. Lancet. 2006;368:338.

10. Stewart JH, Hobbs JB, McCredie MR. Morphologic evidence that analgesic-induced kidney pathology contributes to the progression of tumors of the renal pelvis. Cancer. 1999;86:1576-82.

11. Chou $\mathrm{YH}$, Huang $\mathrm{CH}$. Unusual clinical presentation of upper urothelial carcinoma in Taiwan. Cancer. 1999;85:1342-4.

12. Tan LB, Chen KT, Guo HR. Clinical and epidemiological features of patients with genitourinary tract tumour in a blackfoot disease endemic area of Taiwan. BJU Int. 2008;102:48-54.

13. Yang $\mathrm{MH}$, Chen $\mathrm{KK}$, Yen CC, et al. Unusually high incidence of upper urinary tract urothelial carcinoma in Taiwan. Urology. 2002;59:681-7.

14. Zhang $Z$, Furge KA, Yang XJ, Teh BT, Hansel DE. Comparative gene expression profiling analysis of urothelial carcinoma of the renal pelvis and bladder. BMC Med Genomics. 2010;3:58.

15. Catto JW, Yates DR, Rehman I, et al. Behavior of urothelial carcinoma with respect to anatomical location. J Urol. 2007;177:1715-20.

16. Garrett RH, Grisham CM. Biochemistry. 5th ed. Boston, USA: Cengage Learning; 2013.

17. Radisky D, Muschler J, Bissell MJ. Order and disorder: the role of extracellular matrix in epithelial cancer. Cancer Invest. 2002;20:139-53.

18. Sahai E. Illuminating the metastatic process. Nat Rev Cancer. 2007;7:737-49.

19. Steeg PS. Tumor metastasis: mechanistic insights and clinical challenges. Nat Med. 2006;12:895-904.

20. Calabro NE, Kristofik NJ, Kyriakides TR. Thrombospondin-2 and extracellular matrix assembly. Biochim Biophys Acta. 2014;1840:2396-402.

21. Tokunaga T, Nakamura M, Oshika $Y$, et al. Thrombospondin 2 expression is correlated with inhibition of angiogenesis and metastasis of colon cancer. $\mathrm{Br} \mathrm{J}$ Cancer. 1999;79:354-9.

22. Nakamura M, Oida $Y$, Abe $Y$, et al. Thrombospondin-2 inhibits tumor cell invasion through the modulation of MMP-9 and UPA in pancreatic cancer cells. Mol Med Rep. 2008;1:423-7.

23. Chijiwa $T$, Abe $Y$, Inoue $Y$, et al. Cancerous, but not stromal, thrombospondin-2 contributes prognosis in pulmonary adenocarcinoma. Oncol Rep. 2009;22:279-83.

24. Farrow B, Berger DH, Rowley D. Tumor-derived pancreatic stellate cells promote pancreatic cancer cell invasion through release of thrombospondin-2. J Surg Res. 2009;156:155-60. 
25. Matos AR, Coutinho-Camillo CM, Thuler LC, et al. Expression analysis of thrombospondin 2 in prostate cancer and benign prostatic hyperplasia. Exp Mol Pathol. 2013;94:438-44.

26. Sun $\mathrm{R}, \mathrm{Wu} \mathrm{J}$, Chen $\mathrm{Y}$, et al. Down regulation of Thrombospondin 2 predicts poor prognosis in patients with gastric cancer. Mol Cancer. 2014;13:225.

27. Naumnik W, Ossolińska M, Płońska I, Chyczewska E, Nikliński J. Circulating Thrombospondin-2 and FGF-2 in Patients with Advanced Non-small Cell Lung Cancer: Correlation with Survival. Adv Exp Med Biol. 2015;833:9-14.

28. Lin CY, Lin CY, Chang IW, et al. Low thrombospondin 2 expression is predictive of low tumor regression after neoadjuvant chemoradiotherapy in rectal cancer. Am J Transl Res. 2015;7:2423-32.

29. Riester $\mathrm{M}$, Werner $\mathrm{L}$, Bellmunt $\mathrm{J}$, et al. Integrative analysis of $1 \mathrm{q} 23.3$ copy-number gain in metastatic urothelial carcinoma. Clin Cancer Res. 2014;20:1873-83.

30. Chang IW, Lin VC, Hung CH, et al. GPX2 underexpression indicates poor prognosis in patients with urothelial carcinomas of the upper urinary tract and urinary bladder. World J Urol. 2015;33:1777-89.

31. Kim HS, Piao S, Moon KC, et al. Adjuvant Chemotherapy Correlates with Improved Survival after Radical Cystectomy in Patients with pT3b (Macroscopic Perivesical Tissue Invasion) Bladder Cancer. J Cancer. 2015;6:750-8.

32. Adams JC, Lawler J. The thrombospondins. Int J Biochem Cell Biol. 2004;36:961-8

33. Bornstein P. Thrombospondins as matricellular modulators of cell function. J Clin Invest. 2001;107:929-34.

34. Lawler J, Detmar M. Tumor progression: the effects of thrombospondin-1 and -2. Int J Biochem Cell Biol. 2004;36:1038-45.

35. Chang IW, Liang PI, Li CC, et al. HAS3 underexpression as an indicator of poor prognosis in patients with urothelial carcinoma of the upper urinary tract and urinary bladder. Tumour Biol. 2015;36:5441-50.

36. Chang IW, Lin VC, He HL, et al CDCA5 overexpression is an indicator of poor prognosis in patients with urothelial carcinomas of the upper urinary tract and urinary bladder. Am J Transl Res. 2015;7:710-22.

37. Chang IW, Wu WJ, Wang YH, et al. BCAT1 overexpression is an indicator of poor prognosis in patients with urothelial carcinomas of the upper urinary tract and urinary bladder. Histopathology. 2016;68:520-32

38. Wang $\mathrm{YH}, \mathrm{Wu} \mathrm{WJ}$, Wang WJ, et al. CEBPD amplification and overexpression in urothelial carcinoma: a driver of tumor metastasis indicating adverse prognosis. Oncotarget. 2015;6:31069-84.

39. Chang IW, Wang $\mathrm{YH}, \mathrm{Wu}$ WJ, et al. Necdin overexpression predicts poor prognosis in patients with urothelial carcinomas of the upper urinary tract and urinary bladder. J Cancer. 2016;7:304-13.

40. Ma LJ, Wu WJ, Wang YH, et al. SPOCK1 overexpression confers a poor prognosis in urothelial carcinoma. J Cancer. 2016;7:467-76.

41. Lee $Y Y, W u$ WJ, Huang $C N$, et al. CSF2 overexpression is associated with STAT5 phosphorylation and poor prognosis in patients with urothelial carcinoma. J Cancer. 2016;7:711-21. 